\title{
Firmly nonexpansive mappings and maximally monotone operators: correspondence and duality
}

\author{
Heinz H. Bauschke*, Sarah M. Moffat, and Xianfu Wang ${ }^{\ddagger}$
}

January 24,2011

\begin{abstract}
The notion of a firmly nonexpansive mapping is central in fixed point theory because of attractive convergence properties for iterates and the correspondence with maximal monotone operators due to Minty. In this paper, we systematically analyze the relationship between properties of firmly nonexpansive mappings and associated maximal monotone operators. Dual and self-dual properties are also identified. The results are illustrated through several examples.
\end{abstract}

2010 Mathematics Subject Classification: Primary 47H05, 47H09; Secondary 26B25, 52A41, $90 \mathrm{C} 25$.

Keywords: Banach contraction, convex function, firmly nonexpansive mapping, fixed point, Hilbert space, Legendre function, maximal monotone operator, nonexpansive mapping, paramonotone, proximal map, rectangular, resolvent, subdifferential operator.

*Mathematics, Irving K. Barber School, UBC Okanagan, Kelowna, British Columbia V1V 1V7, Canada. E-mail: heinz.bauschke@ubc.ca.

${ }^{\dagger}$ Mathematics, Irving K. Barber School, UBC Okanagan, Kelowna, British Columbia V1V 1V7, Canada. E-mail: sarah.moffat@ubc.ca.

¥Mathematics, Irving K. Barber School, UBC Okanagan, Kelowna, British Columbia V1V 1V7, Canada. E-mail: shawn. wang@ubc.ca. 


\section{Introduction}

Throughout this paper,

(1) $X$ is a real Hilbert space with inner product $\langle\cdot, \cdot\rangle$ and induced norm $\|\cdot\|$.

Recall that a mapping

$$
T: X \rightarrow X
$$

is firmly nonexpansive if

$$
(\forall x \in X)(\forall y \in X) \quad\|T x-T y\|^{2}+\|(\operatorname{Id}-T) x-(\operatorname{Id}-T) y\|^{2} \leq\|x-y\|^{2},
$$

where Id: $X \rightarrow X: x \mapsto x$ denotes the identity operator. It is clear that if $T$ is firmly nonexpansive, then it is nonexpansive, i.e., Lipschitz continuous with constant 1 ,

$$
(\forall x \in X)(\forall y \in X) \quad\|T x-T y\| \leq\|x-y\| ;
$$

the converse, however, is false (consider - Id). When $T$ is Lipschitz continuous with a constant in $[0,1[$, then we shall refer to $T$ as a Banach contraction.

Returning to firmly nonexpansive mappings, let us provide a useful list of well-known characterizations.

Fact 1.1 (See, e.g., [5, 15, 16].) Let $T: X \rightarrow X$. Then the following are equivalent:

(i) $T$ is firmly nonexpansive.

(ii) $\mathrm{Id}-\mathrm{T}$ is firmly nonexpansive.

(iii) $2 \mathrm{~T}$ - Id is nonexpansive.

(iv) $(\forall x \in X)(\forall y \in X)\|T x-T y\|^{2} \leq\langle x-y, T x-T y\rangle$.

(v) $(\forall x \in X)(\forall y \in X) .0 \leq\langle T x-T y,(I d-T) x-(\mathrm{Id}-T) y\rangle$.

Various problems in the natural sciences and engineering can be converted into a fixed point problem, where the set of desired solutions is the set of fixed points

$$
\text { Fix } T:=\{x \in X \mid x=T x\} .
$$


If $T$ is firmly nonexpansive and Fix $T \neq \varnothing$, then the sequence of iterates

$$
\left(T^{n} x\right)_{n \in \mathbb{N}}
$$

is well known to converge weakly to a fixed point [8] - this is not true for mappings that are merely nonexpansive; consider, e.g., - Id.

Firmly nonexpansive mappings are also important because of their correspondence with maximally monotone operators. Recall that a set-valued operator $A: X \rightrightarrows X$ (i.e., $(\forall x \in X) A x \subseteq X)$ with graph gr $A$ is monotone if

$$
(\forall(x, u) \in \operatorname{gr} A)(\forall(y, v) \in \operatorname{gr} A) \quad\langle x-y, u-v\rangle \geq 0,
$$

and that $A$ is maximally monotone if it is impossible to find a proper extension of $A$ that is still monotone. We write $\operatorname{dom} A:=\{x \in X \mid A x \neq \varnothing\}$ and $\operatorname{ran} A:=A(X)=\bigcup_{x \in X} A x$ for the domain and range of $A$, respectively. Monotone operators play a crucial role in modern nonlinear analysis and optimization; see, e.g., the books [5], [6], [7], [9], [22], [23], [24], [26], [27], and [28].

Prime examples of maximally monotone operators are continuous linear monotone operators and subdifferential operators (in the sense of convex analysis) of functions that are convex, lower semicontinuous, and proper. It will be convenient to set

$$
\left.\left.\Gamma_{0}:=\{f: X \rightarrow]-\infty,+\infty\right] \mid f \text { is convex, lower semicontinuous, and proper }\right\}
$$

See [19], [21], [24], and [5] for background material on convex analysis.

Now let $A: X \rightrightarrows X$ be maximally monotone and denote the associated resolvent by

$$
J_{A}:=(\mathrm{Id}+A)^{-1} .
$$

When $A=\partial f$ for some $f \in \Gamma_{0}$, i.e., $A$ is a subdifferential operator, then we also write $J_{\partial f}=\operatorname{Prox}_{f}$ and, following Moreau [18], we refer to this mapping as the proximal mapping. In his seminal paper [17], Minty observed that $J_{A}$ is in fact a firmly nonexpansive operator from $X$ to $X$ and that, conversely, every firmly nonexpansive operator arises this way:

Fact 1.2 (Minty) (See, e.g., [17] or [13].) Let $T: X \rightarrow X$ be firmly nonexpansive, and let $A: X \rightrightarrows X$ be maximally monotone. Then the following hold.

(i) $B=T^{-1}-$ Id is maximally monotone (and $J_{B}=T$ ). 
(ii) $J_{A}$ is firmly nonexpansive (and $A=J_{A}^{-1}-\mathrm{Id}$ ).

Therefore, the mapping

$$
T \mapsto T^{-1}-\text { Id }
$$

from the set of firmly nonexpansive mappings to the set of maximal monotone operators is a bijection, with inverse

$$
A \mapsto J_{A}=(\mathrm{Id}+A)^{-1} .
$$

Each class of objects has its own duality operation: if $T: X \rightarrow X$ is firmly nonexpansive, then so is the associated dual mapping (see Fact 1.1)

$$
\text { Id }-T \text {; }
$$

note that the dual of the dual is indeed $\mathrm{Id}-(\mathrm{Id}-T)=T$. Analogously, if $A: X \rightrightarrows X$ is maximally monotone, then so is the inverse operator

$$
A^{-1} \text {, }
$$

and we clearly have $\left(A^{-1}\right)^{-1}=A$. Traversing between the two classes and dualizing is commutative as the resolvent identity

$$
\mathrm{Id}=J_{A}+J_{A^{-1}}
$$

shows. We also have the Minty parametrization

$$
\operatorname{gr} A=\left\{\left(J_{A} x,\left(\operatorname{Id}-J_{A}\right) x\right) \mid x \in X\right\},
$$

which provides the bijection $x \mapsto\left(J_{A} x, x-J_{A} x\right)$ from $X$ onto gr $A$, with inverse $(x, u) \mapsto$ $x+u$.

The goal of this paper is threefold. First, we will provide a comprehensive catalogue of corresponding properties of firmly nonexpansive mappings and maximally monotone operators. Second, we shall examine duality of these properties, and also identify those properties that are selfdual, i.e., a property holds for $T$ if and only if the same property holds for $\mathrm{Id}-T$, and a corresponding property holds for $A$ if and only if it holds for $A^{-1}$ in the context of maximally monotone operators. Third, we revisit some of these properties in more specialized settings and present applications of our results to operators occurring in splitting methods, including reflected resolvents.

The paper is organized as follows. In Section 2 , we systematically study properties of firmly nonexpansive mappings and corresponding properties of maximally monotone operators. Section 3 revisits these properties from a duality point of view. In the final Section 4 , we also touch upon corresponding properties of nonexpansive mappings and provide a few possible applications to mappings arising in algorithms.

The notation we employ is standard and as in [5], [19], [21], or [24]. These books provide also background material for notions not explicitly reviewed here. 


\section{Correspondence of Properties}

The main result in this section is the following result, which provides a comprehensive list of corresponding properties of firmly nonexpansive mappings and maximally monotone operators.

Theorem 2.1 Let $T: X \rightarrow X$ be firmly nonexpansive, let $A: X \rightrightarrows X$ be maximally monotone, and suppose that $T=J_{A}$ or equivalently that $A=T^{-1}-\mathrm{Id}$. Then the following hold:

(i) $\operatorname{ran} T=\operatorname{dom} A$.

(ii) $T$ is surjective if and only if $\operatorname{dom} A=X$.

(iii) Id - T is surjective if and only if $A$ is surjective.

(iv) $T$ is injective if and only if $A$ is at most single-valued.

(v) $T$ is an isometry, i.e.,

$$
(\forall x \in X)(\forall y \in X) \quad\|T x-T y\|=\|x-y\|
$$

if and only if there exists $z \in X$ such that $A: x \mapsto z$, in which case $T: x \mapsto x-z$.

(vi) T satisfies

$$
(\forall x \in X)(\forall y \in X) \quad T x \neq T y \Rightarrow\|T x-T y\|^{2}<\langle x-y, T x-T y\rangle
$$

if and only if $A$ is strictly monotone, i.e.,

$$
(\forall(x, u) \in \operatorname{gr} A)(\forall(y, v) \in \operatorname{gr} A) \quad x \neq y \Rightarrow\langle x-y, u-v\rangle>0 .
$$

(vii) $T$ is strictly monotone if and only if $A$ is at most single-valued.

(viii) $T$ is strictly firmly nonexpansive, i.e.,

$$
(\forall x \in X)(\forall y \in X) \quad x \neq y \Rightarrow\|T x-T y\|^{2}<\langle x-y, T x-T y\rangle
$$

if and only if $A$ is at most single-valued and strictly monotone.

(ix) $T$ is strictly nonexpansive, i.e.,

$$
(\forall x \in X)(\forall y \in X) \quad x \neq y \Rightarrow\|T x-T y\|<\|x-y\|
$$

if and only if $A$ is disjointly injective 1 , i.e.,

$$
(\forall x \in X)(\forall y \in X) \quad x \neq y \Rightarrow A x \cap A y=\varnothing .
$$

\footnotetext{
${ }^{1}$ In some of the literature, $A$ is simply referred to as "injective" but this is perhaps a little imprecise: "injectivity of $A$ " could be interpreted as " $[\{x, y\} \subseteq \operatorname{dom} A$ and $x \neq y] \Rightarrow A x \neq A y$ " which is different from what we require here.
} 
(x) $T$ is injective and strictly nonexpansive, i.e.,

$$
(\forall x \in X)(\forall y \in X) \quad x \neq y \Rightarrow 0<\|T x-T y\|<\|x-y\|
$$

if and only if $A$ is at most single-valued and disjointly injective.

(xi) Suppose that $\varepsilon \in] 0,+\infty[$. Then $(1+\varepsilon) T$ is firmly nonexpansive if and only if $A$ is strongly monotone with constant $\varepsilon$, i.e., $A-\varepsilon \mathrm{Id}$ is monotone, in which case $T$ is a Banach contraction with constant $(1+\varepsilon)^{-1}$.

(xii) Suppose that $\gamma \in] 0,+\infty[$. Then $(1+\gamma)(\mathrm{Id}-T)$ is firmly nonexpansive if and only if $A$ is $\gamma$-cocoercive, i.e.,

$$
(\forall(x, u) \in \operatorname{gr} A)(\forall(y, v) \in \operatorname{gr} A) \quad\langle x-y, u-v\rangle \geq \gamma\|u-v\|^{2} .
$$

(xiii) Suppose that $\beta \in] 0,1[$. Then $T$ is a Banach contraction with constant $\beta$ if and only if $A$ satisfies

$$
(\forall(x, u) \in \operatorname{gr} A)(\forall(y, v) \in \operatorname{gr} A) \quad \frac{1-\beta^{2}}{\beta^{2}}\|x-y\|^{2} \leq 2\langle x-y, u-v\rangle+\|u-v\|^{2} .
$$

(xiv) Suppose that $\phi:[0,+\infty[\rightarrow[0,+\infty]$ is increasing and vanishes only at 0 . Then $T$ satisfies

$$
(\forall x \in X)(\forall y \in X) \quad\langle T x-T y,(x-T x)-(y-T y)\rangle \geq \phi(\|T x-T y\|)
$$

if and only if $A$ is uniformly monotone with modulus $\phi$, i.e.,

$$
(\forall(x, u) \in \operatorname{gr} A)(\forall(y, v) \in \operatorname{gr} A) \quad\langle x-y, u-v\rangle \geq \phi(\|x-y\|) .
$$

(xv) T satisfies

$$
(\forall x \in X)(\forall y \in X) \quad\|T x-T y\|^{2}=\langle x-y, T x-T y\rangle \Rightarrow\left\{\begin{array}{l}
T x=T(T x+y-T y) \\
T y=T(T y+x-T x)
\end{array}\right.
$$

if and only if $A$ is paramonotone [10], i.e.,

$$
(\forall(x, u) \in \operatorname{gr} A)(\forall(y, v) \in \operatorname{gr} A) \quad\langle x-y, u-v\rangle=0 \Rightarrow\{(x, v),(y, u)\} \subseteq \operatorname{gr} A .
$$

(xvi) (Bartz et al.) $T$ is cyclically firmly nonexpansive, i.e.,

$$
\sum_{i=1}^{n}\left\langle x_{i}-T x_{i}, T x_{i}-T x_{i+1}\right\rangle \geq 0,
$$

for every set of points $\left\{x_{1}, \ldots, x_{n}\right\} \subseteq X$, where $n \in\{2,3, \ldots\}$ and $x_{n+1}=x_{1}$, if and only if $A$ is a subdifferential operator, i.e., there exists $f \in \Gamma_{0}$ such that $A=\partial f$. 
(xvii) T satisfies

$$
(\forall x \in X)(y \in X) \quad \inf _{z \in X}\langle T x-T z,(y-T y)-(z-T z)\rangle>-\infty
$$

if and only if $A$ is rectangular [23, Definition 31.5] (this is also known as $3^{*}$ monotone), i.e.,

$$
(\forall x \in \operatorname{dom} A)(\forall v \in \operatorname{ran} A) \quad \inf _{(z, w) \in \operatorname{gr} A}\langle x-z, v-w\rangle>-\infty .
$$

(xviii) $T$ is linear if and only if $A$ is a linear relation, i.e., gr $A$ is a linear subspace of $X \times X$.

(xix) $T$ is affine if and only if $A$ is an affine relation, i.e., gr $A$ is an affine subspace of $X \times X$.

(xx) (Zarantonello) $\operatorname{ran} T=$ Fix $T=: C$ if and only if $A$ is a normal cone operator, i.e., $A=$ $\partial \iota_{C}$; equivalently, $T$ is a projection (nearest point) mapping $P_{C}$.

(xxi) $T$ is sequentially weakly continuous if and only if gr $A$ is sequentially weakly closed.

Proof. Let $x, y, u, v$ be in $X$.

(i); Clear.

(ii): This follows from (i),

(iii) Clear from the Minty parametrization (15).

(iv) Assume first that $T$ is injective and that $\{u, v\} \subseteq A x$. Then $\{x+u, x+v\} \subseteq$ $(\operatorname{Id}+A) x$ and hence $x=T(x+u)=T(x+v)$. Since $T$ is injective, it follows that $x+u=$ $x+v$ and hence that $u=v$. Thus, $A$ is at most single-valued. Conversely, let us assume that $T u=T v=x$. Then $\{u, v\} \subseteq(\operatorname{Id}+A) x=x+A x$ and hence $\{u-x, v-x\} \subseteq A x$. Since $A$ is at most single-valued, we have $u-x=v-x$ and so $u=v$. Thus, $T$ is injective.

(v) Assume first that $T$ is an isometry. Then $\|T x-T y\|^{2}=\|x-y\|^{2} \geq\|T x-T y\|^{2}+$ $\|(\operatorname{Id}-T) x-(\operatorname{Id}-T) y\|^{2}$. It follows that there exists $z \in X$ such that $T: w \mapsto w-z$. Thus, $T^{-1}: w \mapsto w+z$. On the other hand, $T^{-1}=\operatorname{Id}+A: w \mapsto w+A w$. Hence $A: w \mapsto z$, as claimed. Conversely, let us assume that there exits $z \in X$ such that $A: w \mapsto z$. Then $\mathrm{Id}+A: w \mapsto w+z$ and hence $T=J_{A}=(\operatorname{Id}+A)^{-1}: w \mapsto w-z$. Thus, $T$ is an isometry.

(vi) Assume first that $T$ satisfies (17), that $\{(x, u),(y, v)\} \subseteq$ gr $A$, and that $x \neq y$. Set $p=x+u$ and $q=y+v$. Then $(x, u)=(T p, p-T p)$ and $(y, v)=(T q, q-T q)$. Since $x \neq y$, it follows that $T p \neq T q$ and therefore that $\|T p-T q\|^{2}<\langle p-q, T p-T q\rangle$ because $T$ satisfies (17). Hence $0<\langle(p-T p)-(q-T q), T p-T q\rangle=\langle u-v, x-y\rangle$. Thus, $A$ is strictly monotone. Conversely, let us assume that $A$ is strictly monotone and that $x=$ $T u \neq T v=y$. Then $\{(x, u-x),(y, v-y)\} \subseteq$ gr $A$. Since $x \neq y$ and $A$ is strictly monotone, 
we have $\langle x-y,(u-x)-(v-y)\rangle>0$, i.e., $\|x-y\|^{2}<\langle x-y, u-v\rangle$, i.e., $\|T u-T v\|^{2}<$ $\langle T v-T u, u-v\rangle$. Thus, $T$ satisfies (17).

(vii) In view of (vi) it suffices to show that $T$ is injective if and only if $T$ is strictly monotone. Assume first that $T$ is injective and that $x \neq y$. Then $T x \neq T y$ and hence $0<\|T x-T y\|^{2} \leq\langle x-y, T x-T y\rangle$. Thus, $T$ is strictly monotone. Conversely, assume that $T$ is strictly monotone and that $x \neq y$. Then $\langle x-y, T x-T y\rangle>0$ and hence $T x \neq T y$. Thus, $T$ is injective.

(viii) Observe that $T$ is strictly firmly nonexpansive if and only if $T$ is injective and $T$ satisfies (17). Thus, the result follows from combining (iv) and (vi).

(ix): Assume first that $T$ is strictly nonexpansive, that $x \neq y$, and to the contrary that $u \in A x \cap A y$. Then $x+u \in(\operatorname{Id}+A) x$ and $y+u \in(\operatorname{Id}+A) y$; equivalently, $T(x+u)=$ $x \neq y=T(y+u)$. Since $T$ is strictly nonexpansive, we have $\|x-y\|=\| T(x+u)-T(y+$ $u)\|<\|(x+u)-(y+u)\|=\| x-y \|$, which is absurd. Thus, $A$ is disjointly injective. Conversely, assume that $A$ is disjointly injective, that $u \neq v$, and to the contrary that $\|T u-T v\|=\|u-v\|$. Since $T$ is firmly nonexpansive, we deduce that $u-T u=v-T v$. Assume that $x=u-T u=v-T v$. Then $T u=u-x$ and $T v=v-x$; equivalently, $u-x \in(\operatorname{Id}+A) u$ and $v-x \in(\operatorname{Id}+A) v$. Thus, $-x \in A u \cap A v$, which contradicts the assumption on disjoint injectivity of $A$.

(x): Combine (iv) and (ix).

(xi)] (See also [5, Proposition 23.11].) Assume first that $(1+\varepsilon) T$ is firmly nonexpansive and that $\{(x, u),(y, v)\} \subseteq$ gr $A$. Then $x=T(x+u)$ and $y=T(y+v)$. Hence, $\langle(x+u)-(y+v), x-y\rangle \geq(1+\varepsilon)\|x-y\|^{2} \Leftrightarrow\langle x-y, u-v\rangle \geq \varepsilon\|x-y\|^{2}$. Thus, $A-\varepsilon \mathrm{Id}$ is monotone. Conversely, assume that $A-\varepsilon \operatorname{Id}$ is monotone and that $\{(x, u),(y, v)\} \subseteq$ $\operatorname{gr} T$. Then $\{(u, x-u),(v, y-v)\} \subseteq \operatorname{gr} A$ and hence $\langle u-v,(x-u)-(y-v)\rangle \geq \varepsilon \| u-$ $v \|^{2}$; equivalently, $\langle x-y, u-v\rangle \geq(1+\varepsilon)\|u-v\|^{2}$. Thus, $(1+\varepsilon) T$ is firmly nonexpansive.

(xii). Applying (xi) to Id $-T$ and $A^{-1}$, we see that $(1+\gamma)(\mathrm{Id}-T)$ is firmly nonexpansive if and only if $A^{-1}-\gamma \mathrm{Id}$ is monotone, which is equivalent to $A$ being $\gamma$-cocoercive.

(xiii), Assume first that $T$ is a Banach contraction with constant $\beta$ and that $\{(x, u),(y, v)\} \subseteq \operatorname{gr} A$. Set $p=x+u$ and $y=y+v$. Then $(x, u)=(T p, p-T p)$, $(y, v)=(T q, q-T q)$, and $\|T p-T q\| \leq \beta\|p-q\|$, i.e.,

$$
\begin{aligned}
\|x-y\|^{2} & \leq \beta^{2}\|(x+u)-(y+v)\|^{2}=\beta^{2}\|(x-y)+(u-v)\|^{2} \\
& =\beta^{2}\left(\|x-y\|^{2}+2\langle x-y, u-v\rangle+\|u-v\|^{2}\right) .
\end{aligned}
$$

Thus, (24) holds. The converse is proved similarly.

(xiv): The equivalence is immediate from the Minty parametrization (15). 
(xv): Assume first that $T$ satisfies (27) and that $\{(x, u),(y, v)\} \subseteq$ gr $A$ with $\langle x-y, u-v\rangle=0$. Set $p=x+u$ and $q=y+v$. Then $(x, u)=(T p, p-T p)$, $(y, v)=(T q, q-T q)$, and $\langle T p-T q,(p-T p)-(q-T q)\rangle=0$, i.e., $\|T p-T q\|^{2}=$ $\langle p-q, T p-T q\rangle$. By (27), $T p=T(T p+q-T q)$ and $T q=T(T q+p-T p)$, i.e., $x=T(x+v)$ and $y=T(y+u)$, i.e., $x+v \in x+A x$ and $y+u \in y+A y$, i.e., $v \in A x$ and $u \in A y$. Thus, $A$ is paramonotone. Conversely, assume that $A$ is paramonotone, that $\|T u-T v\|^{2}=\langle u-v, T u-T v\rangle$, that $x=T u$, and that $y=T v$. Then $\{(x, u-x),(y, v-y)\} \subseteq \operatorname{gr} A$ and $\langle x-y,(u-x)-(v-y)\rangle=0$. Since $A$ is paramonotone, we deduce that $v-y \in A x$ and $u-x \in A y$, i.e., $x-y+v \in(\operatorname{Id}+A) x$ and $y-x+u \in(\operatorname{Id}+A) y$, i.e., $x=T(x-y+v)$ and $y=T(y-x+u)$, i.e., $\mathrm{Tu}=$ $T(T u+v-T v)$ and $T v=T(T v+u-T u)$. Thus, $T$ satisfies (27).

(xvi); This follows from [2, Theorem 6.6].

(xvii); The equivalence is immediate from the Minty parametrization (15).

(xviii) Indeed, $T=J_{A}$ is linear $\Leftrightarrow(A+\mathrm{Id})^{-1}$ is a linear relation $\Leftrightarrow A+\mathrm{Id}$ is a linear relation $\Leftrightarrow A$ is a linear relation (see [11] for more on linear relations).

(xix): This follows from (xviii).

$(\mathrm{xx})$ : This follows from [25, Corollary 2 on page 251].

(xxi): Assume first that $T$ is sequentially weakly continuous. Let $\left(x_{n}, u_{n}\right)_{n \in \mathbb{N}}$ be a sequence in gr $A$ that converges weakly to $(x, u) \in X \times X$. Then $\left(x_{n}+u_{n}\right)_{n \in \mathbb{N}}$ converges weakly to $x+u$. On the other hand, $\mathrm{Id}-T$ is sequentially weakly continuous because $T$ is. Altogether, $\left(x_{n}, u_{n}\right)_{n \in \mathbb{N}}=\left(T\left(x_{n}+u_{n}\right),(\operatorname{Id}-T)\left(x_{n}+u_{n}\right)\right)_{n \in \mathbb{N}}$ converges weakly to $(x, u)=(T(x+u),(\operatorname{Id}-T)(x+u))$. Thus, $(x, u) \in \operatorname{gr} A$ and therefore gr $A$ is sequentially weakly closed. Conversely, let us assume that gr $A$ is sequentially weakly closed. Let $\left(x_{n}\right)_{n \in \mathbb{N}}$ be a sequence in $X$ that is weakly convergent to $x$. Our goal is to show that $T x_{n} \rightarrow T x$. Since $T$ is nonexpansive, the sequence $\left(T x_{n}\right)_{n \in \mathbb{N}}$ is bounded. After passing to a subsequence and relabeling if necessary, we can and do assume that $\left(T x_{n}\right)_{n \in \mathbb{N}}$ converges weakly to some point $y \in X$. Now $\left(T x_{n}, x_{n}-T x_{n}\right)_{n \in \mathbb{N}}$ lies in gr $A$, and this sequence converges weakly to $(y, x-y)$. Since gr $A$ is sequentially weakly closed, it follows that $(y, x-y) \in \operatorname{gr} A$. Therefore, $x-y \in A y \Leftrightarrow x \in(\operatorname{Id}+A) y \Leftrightarrow y=T x$, which implies the result.

Example 2.2 Concerning items (xi) and (xiii) in Theorem 2.1, it is clear that if $A$ is strongly monotone, then $T$ is a Banach contraction. (This result is now part of the folklore; for perhaps the first occurrence see [20, p. 879f] and also [21, Section 12.H] for further results in this direction.) The converse, however, is false: indeed, consider the case $X=\mathbb{R}^{2}$ and set

$$
A=\left(\begin{array}{cc}
0 & -1 \\
1 & 0
\end{array}\right)
$$


Then $(\forall z \in X)\langle z, A z\rangle=0$ so $A$ cannot be strongly monotone. On the other hand,

$$
T=J_{A}=(\mathrm{Id}+A)^{-1}=\frac{1}{2}\left(\begin{array}{cc}
1 & 1 \\
-1 & 1
\end{array}\right)
$$

is linear and $\|T z\|^{2}=\frac{1}{2}\|z\|^{2}$, which implies that $T$ is a Banach contraction with constant $1 / \sqrt{2}$.

Corollary 2.3 Let $A: X \rightarrow X$ be continuous, linear, and maximally monotone. Then the following hold.

(i) If $J_{A}$ is a Banach contraction, then $A$ is (disjointly) injective.

(ii) If $\operatorname{ran} A$ is closed and $A$ is (disjointly) injective, then $J_{A}$ is a Banach contraction.

Proof. The result is trivial if $X=\{0\}$ so we assume that $X \neq\{0\}$.

(i) Assume that $J_{A}$ is a Banach contraction, say with constant $\beta \in[0,1[$. If $\beta=0$, then $J_{A} \equiv 0 \Leftrightarrow A=N_{\{0\}}$, which contradicts the single-valuedness of $A$. Thus, $0<\beta<1$. By Theorem 2.1](xiii),

$$
(\forall x \in X)(\forall y \in X) \quad \frac{1-\beta^{2}}{\beta^{2}}\|x-y\|^{2} \leq 2\langle x-y, A x-A y\rangle+\|A x-A y\|^{2} .
$$

If $x \neq y$, then the left side of (35) is strictly positive, which implies that $A x \neq A y$. Thus, $A$ is (disjointly) injective.

(ii) : Let us assume that $\operatorname{ran} A$ is closed and that $A$ is (disjointly) injective. Then $\operatorname{ker} A=$ $\{0\}$ and hence, by e.g. [12, Theorem 8.18], there exists $\rho \in] 0,+\infty[$ such that $(\forall z \in X)$ $\|A z\| \geq \rho\|z\|$. Thus, $(\forall z \in X)\|A z\|^{2}-\rho^{2}\|z\| \geq 0$. Set $\beta=1 / \sqrt{1+\rho^{2}}$. Then $\rho^{2}=$ $\left(1-\beta^{2}\right) / \beta^{2}$ and hence

$(\forall x \in X)(\forall y \in X) \quad \frac{1-\beta^{2}}{\beta^{2}}\|x-y\|^{2} \leq\|A x-A y\|^{2} \leq 2\langle x-y, A x-A y\rangle+\|A x-A y\|^{2}$.

Again by Theorem 2.1][xiii), $J_{A}$ is a Banach contraction with constant $\left.\beta \in\right] 0,1[$.

In item[ii) of Corollary 2.3, the assumption that $\operatorname{ran} A$ is closed is critical:

Example 2.4 Suppose that $X=\ell_{2}$, the space of square-summable sequences, i.e., $x=$ $\left(x_{n}\right) \in X$ if and only if $\sum_{n=1}^{\infty}\left|x_{n}\right|^{2}<+\infty$, and set

$$
A: X \rightarrow X:\left(x_{n}\right) \mapsto\left(\frac{1}{n} x_{n}\right) .
$$


Then $A$ is continuous, linear, maximally monotone, and $\operatorname{ran} A$ is a dense, proper subspace of $X$ that is not closed. The resolvent $T=J_{A}$ is

$$
T: X \rightarrow X:\left(x_{n}\right) \mapsto\left(\frac{n}{n+1} x_{n}\right) .
$$

Now denote the $n^{\text {th }}$ unit vector in $X$ by $\mathbf{e}_{n}$. Then $\left\|T \mathbf{e}_{n}-T 0\right\|=\frac{n}{n+1}\left\|\mathbf{e}_{n}-0\right\|$. Since $\frac{n}{n+1} \rightarrow 1$, it follows that $T$ is not a Banach contraction.

When $A$ is a subdifferential operator, then it is impossible to get the behavior witnessed in Example 2.2.

Proposition 2.5 Let $f \in \Gamma_{0}$ and let $\left.\varepsilon \in\right] 0,+\infty\left[\right.$. Then $(1+\varepsilon)$ Prox $_{f}$ is firmly nonexpansive if and only if $\operatorname{Prox}_{f}$ is a Banach contraction with constant $(1+\varepsilon)^{-1}$.

Proof. Set $\beta=(1+\varepsilon)^{-1}$. It is clear that if $(1+\varepsilon) \operatorname{Prox}_{f}$ is firmly nonexpansive, then $(1+\varepsilon) \operatorname{Prox}_{f}$ is nonexpansive and hence $\operatorname{Prox}_{f}$ is a Banach contraction with constant $\beta$. Conversely, assume that $\operatorname{Prox}_{f}$ is a Banach contraction with constant $\beta$. Since $\operatorname{Prox}_{f}$ is the Fréchet gradient mapping of the continuous convex function $f^{*} \square \frac{1}{2}\|\cdot\|^{2}: X \rightarrow \mathbb{R}$, the Baillon-Haddad theorem (see [1] and also [4] for recent results) guarantees that $\beta^{-1}$ Prox $_{f}$ is firmly nonexpansive.

Remark 2.6 If $n=2$, then (29) reduces to

$$
\left\langle x_{1}-T x_{1}, T x_{1}-T x_{2}\right\rangle+\left\langle x_{2}-T x_{2}, T x_{2}-T x_{1}\right\rangle \geq 0,
$$

i.e., to firm nonexpansiveness of $T$ (see Fact 1.1][(v)]).

\section{Duality and Self-Duality}

In the introduction, we pointed out that there is a natural duality for firmly nonexpansive mappings and maximally monotone operators; namely, $T \mapsto \mathrm{Id}-T$ and $A \mapsto A^{-1}$, respectively. Thus, every property considered in Section 2 has a dual property. We have considered all dual properties and we shall explicitly single those out that we found to have simple and pleasing descriptions. Among these properties, those that are "selfdual", i.e., the property is identical to its dual property, stand out even more. Let us begin my making these ideas more precise.

Definition 3.1 (dual and self-dual properties) Let $(p)$ and $\left(p^{*}\right)$ be properties for firmly nonexpansive mappings defined on $X$. If, for every firmly nonexpansive mapping $T: X \rightarrow X$,

$$
T \text { satisfies }(p) \text { if and only if } \mathrm{Id}-T \text { satisfies }\left(p^{*}\right),
$$


then $\left(p^{*}\right)$ is dual to $(p)$, and hence $(p)$ is dual to $\left(p^{*}\right)$. If $(p)=\left(p^{*}\right)$, we say that $(p)$ is selfdual. Analogously, let $(q)$ and $\left(q^{*}\right)$ be properties of maximally monotone operators defined on X. If

$$
\text { A satisfies ( } q) \text { if and only if } A^{-1} \text { satisfies }\left(q^{*}\right)
$$

for every maximally monotone operator $A: X \rightrightarrows X$, then $\left(q^{*}\right)$ is dual to $(q)$, and hence $(q)$ is dual to $\left(q^{*}\right)$. If $(q)=\left(q^{*}\right)$, we say that $(q)$ is self-dual.

We start with a simple example to illustrate these ideas. It appears that here there is not natural dual property to surjectivity of $T$ (except for the obvious "Id $-(\mathrm{Id}-T)$ is surjective", but this is not illuminating).

Theorem 3.2 Let $T: X \rightarrow X$ be firmly nonexpansive, let $A: X \rightrightarrows X$ be maximally monotone, and suppose that $T=J_{A}$ or equivalently that $A=T^{-1}-\mathrm{Id}$. Then the following are equivalent:

(i) $T$ is surjective.

(ii) A has full domain.

(iii) $A^{-1}$ is surjective.

Thus for maximally monotone operators, surjectivity and full domain are properties that are dual to each other. These properties are not self-dual: e.g., $A=0$ has full domain while $A^{-1}=\partial \iota_{\{0\}}$ does not.

Proof. (i) $\Leftrightarrow$ (ii) Theorem 2.1[(ii), (ii) $\Leftrightarrow$ (iii), Obvious.

Theorem 3.3 Let $T: X \rightarrow X$ be firmly nonexpansive, let $A: X \rightrightarrows X$ be maximally monotone, and suppose that $T=J_{A}$ or equivalently that $A=T^{-1}-\mathrm{Id}$. Then the following are equivalent:

(i) $T$ is strictly nonexpansive.

(ii) $A$ is disjointly injective.

(iii) $\mathrm{Id}-T$ is injective.

(iv) $A^{-1}$ is at most single-valued.

Thus for firmly nonexpansive mappings, strict nonexpansiveness and injectivity are dual to each other; and correspondingly for maximally monotone operators disjoint injectivity and at most single-valuedness are dual to each other. These properties are not self-dual: e.g., $T \equiv 0$ is strictly nonexpansive, but Id $-T=\mathrm{Id}$ is not; correspondingly, $A=\partial \iota_{\{0\}}$ is disjointly injective but $A^{-1}=0$ is not. 
Proof. We know that (i) $\Leftrightarrow$ (ii) by Theorem 2.1(ix). We also know that (iii) $\Leftrightarrow$ (iv) by Theorem 2.1](iv) (applied to $A^{-1}$ and Id $-T$ ). It thus suffices to show that (ii) $\Leftrightarrow($ (iv), Assume first that $A$ is disjointly injective and that $\{x, y\} \subseteq A^{-1} u$. Then $u \in A x \cap A y$. Since $A$ is disjointly injective, we have $x=y$. Thus, $A^{-1}$ is at most single-valued. Conversely, assume that $A^{-1}$ is at most single-valued and that $u \in A x \cap A y$. Then $\{x, y\} \subseteq A^{-1} u$ and so $x=y$. It follows that $A$ is disjointly injective.

Theorem 3.4 Let $\mathrm{T}: X \rightarrow X$ be firmly nonexpansive, let $A: X \rightrightarrows X$ be maximally monotone, and suppose that $T=J_{A}$ or equivalently that $A=T^{-1}-\mathrm{Id}$. Then the following are equivalent:

(i) T satisfies (17).

(ii) A is strictly monotone.

(iii) Id $-T$ satisfies

$$
\begin{aligned}
(\forall x \in X)(\forall y \in X) \quad(\operatorname{Id}-(\mathrm{Id}-T)) x \neq(\mathrm{Id}-(\mathrm{Id}-T)) y \\
\quad \Rightarrow\|(\operatorname{Id}-T) x-(\mathrm{Id}-T) y\|^{2}<\langle x-y,(\mathrm{Id}-\mathrm{T}) x-(\mathrm{Id}-T) y\rangle
\end{aligned}
$$

(iv) $A^{-1}$ satisfies

$$
\left(\forall(x, u) \in \operatorname{gr} A^{-1}\right)\left(\forall(y, v) \in \operatorname{gr} A^{-1}\right) \quad u \neq v \Rightarrow\langle x-y, u-v\rangle>0 .
$$

Thus for firmly nonexpansive mappings, properties (17) and (42) are dual to each other; and correspondingly for maximally monotone operators strict monotonicity and (43) are dual to each other. These properties are not self-dual: e.g., $T=0$ trivially satisfies (17), but $\mathrm{Id}-0=\mathrm{Id}$ does not.

Proof. (i) $\Leftrightarrow$ (ii), Theorem 2.1](vi), (i) $\Leftrightarrow$ (iii), Indeed, (42) and (17) are equivalent as is easily seen by expansion and rearranging. (ii) $\Leftrightarrow$ (iv), Clear.

Theorem 3.5 (self-duality of strict firm nonexpansiveness) Let $T: X \rightarrow X$ be firmly nonexpansive, let $A: X \rightrightarrows X$ be maximally monotone, and suppose that $T=J_{A}$ or equivalently that $A=T^{-1}-\mathrm{Id}$. Then the following are equivalent:

(i) $T$ is strictly firmly nonexpansive.

(ii) $A$ is at most single-valued and strictly monotone.

(iii) $\mathrm{Id}-\mathrm{T}$ is strictly firmly nonexpansive.

(iv) $A^{-1}$ is at most single-valued and strictly monotone. 
Consequently, strict firm nonexpansive is a self-dual property for firmly nonexpansive mappings; correspondingly, being both strictly monotone and at most single-valued is self-dual for maximally monotone operators.

Proof. Note that $T$ is strictly firmly nonexpansive if and only if

$$
(\forall x \in X)(\forall y \in X) \quad x \neq y \Rightarrow 0<\langle T x-T y,(\operatorname{Id}-T) x-(\operatorname{Id}-T) y\rangle,
$$

which is obviously self-dual. In view of Theorem 2.1](viii), the corresponding property for $A$ is being both at most single-valued and strictly monotone.

Theorem 3.5 illustrates the technique of obtaining self-dual properties by fusing any property and its dual. Here is another example of this type.

Theorem 3.6 (self-duality of strict nonexpansiveness and injectivity) Let $T: X \rightarrow X$ be firmly nonexpansive, let $A: X \rightrightarrows X$ be maximally monotone, and suppose that $T=J_{A}$ or equivalently that $A=T^{-1}-\mathrm{Id}$. Then the following are equivalent:

(i) $T$ is strictly nonexpansive and injective.

(ii) $A$ is at most single-valued and disjointly injective.

(iii) $\mathrm{Id}-\mathrm{T}$ is strictly nonexpansive and injective.

(iv) $A^{-1}$ is at most single-valued and disjointly injective.

Consequently, being both strictly nonexpansive and injective is a self-dual property for firmly nonexpansive mappings; correspondingly, being both disjointly injective and at most single-valued is self-dual for maximally monotone operators.

Proof. Clear from Theorem 3.3](x),

Remark 3.7 Some comments regarding Theorem 3.5 and Theorem 3.6 are in order.

(i) Arguing directly (or by using the characterization with monotone operators via Theorem 2.1), it is easy to verify the implication

(45) $T$ is strictly firmly nonexpansive $\Rightarrow T$ is injective and strictly nonexpansive.

(ii) The converse of implication (45) is false in general: e.g., suppose that $X=\mathbb{R}^{2}$, and let $A$ denote the counter-clockwise rotation by $\pi / 2$, which we utilized already in (33). Clearly, $A$ is a linear single-valued maximally monotone operator that is (disjointly) injective, but $A$ is not strictly monotone. Accordingly, $T=J_{A}$ is linear, injective and strictly nonexpansive, but not strictly firmly nonexpansive. 
(iii) In striking contrast, we shall see shortly (in Corollary 3.9 below) that when $X$ is finite-dimensional and $T=J_{A}$ is a proximal mapping (i.e., $A$ is a subdifferential operator), then the converse implication of (45) is true. We shall write Prox $f$ for $J_{\partial f}$ when $f \in \Gamma_{0}$.

Lemma 3.8 Suppose that $X$ is finite-dimensional and let $f \in \Gamma_{0}$. Then the following are equivalent:

(i) $\partial f$ is disjointly injective.

(ii) $(\partial f)^{-1}=\partial f^{*}$ is at most single-valued.

(iii) $f^{*}$ is essentially smooth.

(iv) $f$ is essentially strictly convex.

(v) $\partial f$ is strictly monotone.

(vi) $\operatorname{Prox}_{f}$ is strictly nonexpansive.

(vii) $(\forall x \in X)(\forall y \in X) \operatorname{Prox}_{f} x \neq \operatorname{Prox}_{f} y$ $\Rightarrow\left\|\operatorname{Prox}_{f} x-\operatorname{Prox}_{f} y\right\|^{2}<\left\langle x-y, \operatorname{Prox}_{f} x-\operatorname{Prox}_{f} y\right\rangle$.

Proof. "(i) $\Leftrightarrow($ ii)]": Theorem 3.3. "[(ii) $\Leftrightarrow$ (iii)": [19, Theorem 26.1]. "(iii) $\Leftrightarrow($ (iv)]": [19, The-

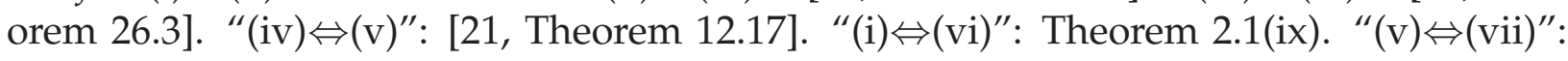
Theorem 2.1][(vi),

Lemma 3.8 admits a dual counterpart that contains various characterizations of essential smoothness. The following consequence of these characterizations is also related to Remark 3.7.

Corollary 3.9 (Legendre self-duality) Suppose that $X$ is finite-dimensional and let $f \in \Gamma_{0}$. Then the following are equivalent:

(i) $\partial f$ is disjointly injective and at most single-valued.

(ii) $\partial f$ is strictly monotone and at most single-valued.

(iii) $f$ is Legendre.

(iv) Prox $_{f}$ is strictly firmly nonexpansive.

\footnotetext{
${ }^{2}$ See [19, Chapter 26] and [21, Section 12.C] for more on functions that are essentially smooth, essentially strictly convex, or of Legendre type.
} 
(v) Prox $_{f}$ is strictly nonexpansive and injective.

(vi) $\partial f^{*}$ is disjointly injective and at most single-valued.

(vii) $\partial f^{*}$ is strictly monotone and at most single-valued.

(viii) $f^{*}$ is Legendre.

(ix) $\operatorname{Prox}_{f^{*}}$ is strictly firmly nonexpansive.

(x) $\operatorname{Prox}_{f^{*}}$ is strictly nonexpansive and injective.

Remark 3.10 When $X$ is infinite-dimensional, the results become more technical and additional assumptions are required due to subtleties of Legendre functions that do not occur in finite-dimensional settings; see [3] and [6].

Remark 3.11 (strong monotonicity and cocoercivity) Concerning Theorem 2.11(xi) \& (xii), we note in passing that strong monotonicity is not self-dual: indeed, $A=\partial \iota_{\{0\}}$ is strongly monotone, but $A^{-1} \equiv 0$ is not. This example also shows that uniform monotonicity is not self-dual. The property dual to strong monotonicity is cocoercivity (which is also known the more accurate name inverse strong monotonicity).

Theorem 3.12 (self-duality of paramonotonicity) Let $A: X \rightrightarrows X$ be maximally monotone, let $T: X \rightarrow X$ be firmly nonexpansive, and suppose that $T=J_{A}$ or equivalently that $A=$ $T^{-1}$ - Id. Then $A$ is paramonotone if and only if $A^{-1}$; consequently, $T$ satisfies (27) if and only if Id $-T$ satisfies (27) (with $T$ replaced by $\mathrm{Id}-T$ ). Consequently, being paramonotone is a is a selfdual property for maximally monotone operators; correspondingly, satisfying (27) is a self-dual property for firmly nonexpansive mappings.

Proof. Self-duality is immediate from the definition of paramonotonicity, and the corresponding result for firmly nonexpansive mappings follows from Theorem 2.1](xv).

\section{Theorem 3.13 (self-duality of cyclical firm nonexpansiveness and cyclical monotonicity)}

Let $T: X \rightarrow X$ be firmly nonexpansive, let $A: X \rightrightarrows X$ be maximally monotone, let $f \in \Gamma_{0}$, and suppose that $T=J_{A}$ or equivalently that $A=T^{-1}-\mathrm{Id}$. Then the following are equivalent:

(i) $T$ is cyclically firmly nonexpansive.

(ii) A is cyclically monotone.

(iii) $A=\partial f$.

(iv) Id - T is cyclically firmly nonexpansive. 
(v) $A^{-1}$ is cyclically monotone.

(vi) $A^{-1}=\partial f^{*}$.

Consequently, cyclic firm nonexpansiveness is a self-dual property for firmly nonexpansive mappings; correspondingly, cyclic monotonicity is is a self-dual property for maximally monotone operators.

Proof. The fact that cyclically maximal monotone operators are subdifferential operators is due to Rockafellar and well known, as is the identity $(\partial f)^{-1}=\partial f^{*}$. The result thus follows from Theorem 2.1](xvi),

Theorem 3.14 (self-duality of rectangularity) Let $T: X \rightarrow X$ be firmly nonexpansive, let $A: X \rightrightarrows X$ be maximally monotone, and suppose that $T=J_{A}$ or equivalently that $A=T^{-1}-\mathrm{Id}$. Then the following are equivalent:

(i) T satisfies (30).

(ii) $A$ is rectangular.

(iii) $\mathrm{Id}-\mathrm{T}$ satisfies (30).

(iv) $A^{-1}$ is rectangular.

Consequently, rectangularity is a self-dual property for maximally monotone operators; correspondingly, (30) is a self-dual property for firmly nonexpansive mappings.

Proof. It is obvious from the definition that either property is self-dual; the equivalences thus follow from Theorem 2.1](xvii),

Theorem 3.15 (self-duality of linearity) Let $T: X \rightarrow X$ be firmly nonexpansive, let $A: X \rightrightarrows$ $X$ be maximally monotone, and suppose that $T=J_{A}$ or equivalently that $A=T^{-1}-\mathrm{Id}$. Then the following are equivalent:

(i) $\mathrm{T}$ is linear.

(ii) A is a linear relation.

(iii) $\mathrm{Id}-\mathrm{T}$ is linear.

(iv) $A^{-1}$ is a linear relation. 
Consequently, linearity is a self-dual property for firmly nonexpansive mappings; correspondingly, being a linear relation is a self-dual property for maximally monotone operators.

Proof. It is clear that $T$ is linear if and only if $\mathrm{Id}-T$ is; thus, the result follows from Theorem 2.1](xviii),

Theorem 3.16 (self-duality of affineness) Let $T: X \rightarrow X$ be firmly nonexpansive, let $A: X \rightrightarrows X$ be maximally monotone, and suppose that $T=J_{A}$ or equivalently that $A=T^{-1}-\mathrm{Id}$. Then the following are equivalent:

(i) $T$ is affine.

(ii) $A$ is an affine relation.

(iii) $\mathrm{Id}-\mathrm{T}$ is affine.

(iv) $A^{-1}$ is an affine relation.

Consequently, affineness is a self-dual property for firmly nonexpansive mappings; correspondingly, being an affine relation is is a self-dual property for maximally monotone operators.

Proof. It is clear that $T$ is affine if and only if $\mathrm{Id}-T$ is; therefore, the result follows from Theorem 2.1](xix).

Remark 3.17 (projection) Concerning Theorem 2.] [ (xx), we note in passing that being a projection is not a self-dual: indeed, suppose that $X \neq\{0\}$ and let $T$ be the projection onto the closed unit ball. Then Id $-T$ is not a projection since $\operatorname{Fix}(\operatorname{Id}-T)=\{0\} \varsubsetneqq X=$ $\operatorname{ran}(\operatorname{Id}-T)$.

Theorem 3.18 (self-duality of sequential weak continuity) Let $T: X \rightarrow X$ be firmly nonexpansive, let $A: X \rightrightarrows X$ be maximally monotone, and suppose that $T=J_{A}$ or equivalently that $A=T^{-1}-\mathrm{Id}$. Then the following are equivalent:

(i) $T$ is sequentially weakly continuous.

(ii) gr $A$ is sequentially weakly closed.

(iii) Id $-T$ is sequentially weakly continuous.

(iv) $A^{-1}$ is sequentially weakly closed.

Consequently, sequential weak continuity is a self-dual property for firmly nonexpansive mappings; correspondingly, having a sequentially weakly closed graph is a self-dual property for maximally monotone operators. 
Proof. Since Id is weakly continuous, it is clear that $T$ is sequentially weakly continuous if and only if $\mathrm{Id}-\mathrm{T}$ is; thus, the result follows from Theorem 2.1] (xxi),

We end this section by listing all self-dual properties encountered so far.

Remark 3.19 Let $T: X \rightarrow X$ be firmly nonexpansive, let $A: X \rightrightarrows X$ be maximally monotone, and suppose that $T=J_{A}$ or equivalently that $A=T^{-1}-\mathrm{Id}$. Then the following properties are self-dual:

(i) $T$ is strictly firmly nonexpansive (Theorem 3.5).

(ii) $T$ is strictly nonexpansive and injective (Theorem 3.6).

(iii) $A$ is paramonotone (Theorem 3.12).

(iv) $A$ is a subdifferential operator (Theorem 3.13).

(v) $A$ is rectangular (Theorem 3.14).

(vi) $T$ is linear (Theorem 3.15).

(vii) $T$ is affine (Theorem 3.16).

(viii) $T$ is sequentially weakly continuous (Theorem 3.18).

\section{Nonexpansive Mappings}

In the previous two sections, we have extensively utilized the the correspondence between firmly nonexpansive mappings and maximally monotone operators. However, Fact 1.1 provides another correspondence, namely with nonexpansive mappings. Indeed,

$T$ is firmly nonexpansive if and only if $N=2 T-$ Id is nonexpansive.

Note that $N$ is also referred to as a reflected resolvent. The corresponding dual of $N$ within the set of nonexpansive mappings is, very elegantly, simply

$$
-N \text {. }
$$

Thus, all results have counterparts formulated for nonexpansive mappings. These counterparts are most easily derived from the firmly nonexpansive formulation, by simply replacing $T$ by $\frac{1}{2} \mathrm{Id}+\frac{1}{2} N$. This makes the proofs fairly straightforward and we accordingly omit them. In the following, we list those cases where the corresponding nonexpansive formulation turned out to be simple and elegant. 
Theorem 4.1 (strict firm nonexpansiveness) Let $T: X \rightarrow X$ be firmly nonexpansive, let $N: X \rightarrow X$ be nonexpansive, and suppose that $N=2 T-I d$. Then $T$ is strictly firmly nonexpansive if and only if $N$ is strictly nonexpansive.

\section{Remark 4.2}

(i) Of course, from Theorem 3.5, we know that strict firm nonexpansiveness is a selfdual property. This can also be seen within the realm of nonexpansive mappings since $N$ is strictly nonexpansive if and only if $-N$ is.

(ii) Furthermore, combining Theorem 3.5 with Theorem 4.1 yields the following: a maximally monotone operator $A$ is at most single-valued and strictly monotone if and only if its reflected resolvent $2 J_{A}-\mathrm{Id}$ is strictly nonexpansive. This characterization was observed by Rockafellar and Wets; see [21, Proposition 12.11].

(iii) In passing, we note that when $X$ is finite-dimensional, the iterates of a strictly nonexpansive mapping converge to the unique fixed point (assuming it exists). For this and more, see, e.g., [14].

The next result pertains to Theorem 2.1](xi),

Theorem 4.3 (strong monotonicity) Let $A: X \rightrightarrows X$ be maximally monotone, let $N: X \rightarrow X$ be nonexpansive, suppose that $N=2 J_{A}-\operatorname{Id}$ and that $\left.\varepsilon \in\right] 0,+\infty[$. Then $A$ is strongly monotone with constant $\varepsilon$ if and only if $\varepsilon \mathrm{Id}+(1+\varepsilon) N$ is nonexpansive.

Theorem 4.4 (reflected resolvent as Banach contraction) Let $A: X \rightrightarrows X$ be maximally monotone, let $T: X \rightarrow X$ be firmly nonexpansive, and let $N: X \rightarrow X$ be nonexpansive. Suppose that $T=J_{A}$, that $N=2 T-\mathrm{Id}$, and that $\beta \in[0,1]$. Then the following are equivalent:

(i) $(\forall(x, u) \in \operatorname{gr} A)(\forall(y, v) \in \operatorname{gr} A)$

$\left(1-\beta^{2}\right)\left(\|x-y\|^{2}+\|u-v\|^{2}\right) \leq 2\left(1+\beta^{2}\right)\langle x-y, u-v\rangle$

(ii) $(\forall x \in X)(\forall y \in X)\left(1-\beta^{2}\right)\|x-y\|^{2} \leq 4\langle T x-T y,(I d-T) x-($ Id $-T) y\rangle$

(iii) $(\forall x \in X)(\forall y \in X)\|N x-N y\| \leq \beta\|x-y\|$.

Proof. In view of the Minty parametrization (see (15)), item (i) is equivalent to

$$
\begin{aligned}
(\forall x \in X)(\forall y \in X) \quad\left(1-\beta^{2}\right)\left(\|T x-T y\|^{2}+\|(x-T x)-(y-T y)\|^{2}\right) \\
\leq \\
\leq 2\left(1+\beta^{2}\right)\langle T x-T y,(x-T x)-(y-T y)\rangle .
\end{aligned}
$$

Simple algebraic manipulations show that (48) is equivalent to (ii), which in turn is equivalent to (iii), 
It is clear that the properties (i) (iii) in Theorem 4.4 are self-dual (for fixed $\beta$ ). The following result is a simple consequence.

Corollary 4.5 (self-duality of reflected resolvents that are Banach contractions) Let $A: X \rightrightarrows$ $X$ be maximally monotone, let $T: X \rightarrow X$ be firmly nonexpansive, let $N: X \rightarrow X$ be nonexpansive, and suppose that $T=J_{A}$ and $N=2 T-I d$. Then the following are equivalent:

(i) $\inf \left\{\frac{\langle x-y, u-v\rangle}{\|x-y\|^{2}+\|u-v\|^{2}} \mid\{(x, u),(y, v)\} \subseteq \operatorname{gr} A\right.$ and $\left.(x, u) \neq(y, v)\right\}>0$.

(ii) $\inf \left\{\frac{\langle T x-T y,(\operatorname{Id}-T) x-(\operatorname{Id}-T) y\rangle}{\|x-y\|^{2}} \mid\{x, y\} \subseteq X\right.$ and $\left.x \neq y\right\}>0$.

(iii) $N$ is a Banach contraction.

Furthermore, these properties are self-dual for their respective classes of operators.

Remark 4.6 Precisely when $A: x \mapsto x-z$ for some fixed vector $z \in X$, we compute $T: x \mapsto(x+z) / 2$ and therefore we reach the extreme case of Corollary 4.5 where $N: x \mapsto$ $z$ is a Banach contraction with constant 0 .

Corollary 4.7 Let $A: X \rightrightarrows X$ be maximally monotone, let $T: X \rightarrow X$ be firmly nonexpansive, let $N: X \rightarrow X$ be nonexpansive, and suppose that $T=J_{A}$ and $N=2 T-I d$. Then the following are equivalent:

(i) Both $A$ and $A^{-1}$ are strongly monotone.

(ii) There exists $\gamma \in] 1,+\infty[$ such that both $\gamma T$ and $\gamma(\mathrm{Id}-T)$ are firmly nonexpansive.

(iii) $N$ is a Banach contraction.

Proof. Let us assume that $A$ and $A^{-1}$ are both strongly monotone; equivalently, there exists $\varepsilon \in] 0,+\infty\left[\right.$ such that $A-\varepsilon \operatorname{Id}$ and $A^{-1}-\varepsilon \operatorname{Id}$ are monotone. Let $\{(x, u),(y, v)\} \subseteq$ gr $A$. Then $\{(u, x),(v, y)\} \subseteq$ gr $A^{-1}$ and

$$
\langle x-y, u-v\rangle \geq \varepsilon\|x-y\|^{2} \text { and }\langle u-v, x-y\rangle \geq \varepsilon\|u-v\|^{2} .
$$

Adding these inequalities yields $2\langle x-y, u-v\rangle \geq \varepsilon\left(\|x-y\|^{2}+\|u-v\|^{2}\right)$. Thus, item (i) of Corollary 4.5 holds. Conversely, if item[(i) of Corollary 4.5 holds, then both $A$ and $A^{-1}$ are strongly monotone. Therefore, by Corollary 4.5, (i) and (iii) are equivalent. Finally, in view of Theorem 2.1[(xi), we see that [i)] and (ii)] are also equivalent.

Additional characterizations are available for subdifferential operators: 
Proposition 4.8 Let $f \in \Gamma_{0}$. Then the following are equivalent:

(i) $f$ and $f^{*}$ are strongly convex.

(ii) $f$ and $f^{*}$ are everywhere differentiable, and both $\nabla f$ and $\nabla f^{*}$ are Lipschitz continuous.

(iii) $\operatorname{Prox}_{f}$ and Id - Prox $f$ are Banach contractions.

(iv) 2 Prox $_{f}$ - Id is a Banach contraction.

Proof. It is well known that for functions, strong convexity is equivalent to strong monotonicity of the subdifferential operators; see, e.g., [5, Example 22.3]. In view of Proposition 2.5 and Corollary 4.7 we obtain the equivalence of items (i), (iii), and (iv). Finally, the equivalence of (i) and (ii) follows from [4, Theorem 2.1].

We now turn to linear relations.

Proposition 4.9 Let $A: X \rightarrow X$ be a maximally monotone linear relation. Then the following are equivalent:

(i) Both $A$ and $A^{-1}$ are strongly monotone.

(ii) $A$ is a continuous surjective linear operator on $X$ and $\inf _{z \in X \backslash\{0\}} \frac{\langle z, A z\rangle}{\|z\|^{2}+\|A z\|^{2}}>0$.

(iii) $2 J_{A}-$ Id is a Banach contraction.

If $X$ is finite-dimensional, then (i)-(iii) are also equivalent to

(iv) $A: X \rightarrow X$ satisfies $(\forall z \in X \backslash\{0\})\langle z, A z\rangle>0$.

Proof. '(i) $\Leftrightarrow($ iii) $]$ ': Clear from Corollary 4.7

“(i) $\Rightarrow$ (ii)]": By, e.g. [5, Example 22.9(iii)], $A$ and $A^{-1}$ are single-valued surjective operators with full domain. Since $A$ and $A^{-1}$ are linear, [5, Corollary 21.19] implies that $A$ and $A^{-1}$ are continuous. Thus, (ii) holds.

“(i) $\Leftrightarrow$ (ii)": (ii) implies that item (i) of Corollary 4.5 holds. Thus, (i) follows from Corollary 4.5 and Corollary 4.7

'[ii) $\Rightarrow$ (iv)]": Clear. 
"(ii) $\Leftrightarrow$ (iv) $]$ ": Since $A$ is injective and $X$ is finite-dimensional, $A$ is bijective and continuous. To see that the infimum in item (ii) is strictly positive, note that we may take the infimum over the unit sphere, which is a compact subset of $X$.

We shall conclude this paper with some comments regarding prototypical applications of the above results to splitting methods (see also [5] for further information and various variants). Here is a technical lemma, which is part of the folklore, and whose simple proof we omit.

Lemma 4.10 Let $T_{1}, \ldots, T_{n}$ be finitely many nonexpansive mappings from $X$ to $X$, and let $\lambda_{1}, \ldots, \lambda_{n}$ be in $\left.] 0,1\right]$ such that $\lambda_{1}+\cdots+\lambda_{n}=1$. Then the following hold:

(i) The composition $T_{1} T_{2} \cdots T_{n}$ is nonexpansive.

(ii) The convex combination $\lambda_{1} T_{1}+\cdots+\lambda_{n} T_{n}$ is nonexpansive.

(iii) If some $T_{i}$ is strictly nonexpansive, then $T_{1} T_{2} \cdots T_{n}$ is strictly nonexpansive.

(iv) If some $T_{i}$ is strictly nonexpansive, then $\lambda_{1} T_{1}+\cdots+\lambda_{n} T_{n}$ is strictly nonexpansive.

(v) If some $T_{i}$ is a Banach contraction, then $T_{1} T_{2} \cdots T_{n}$ is a Banach contraction.

(vi) If some $T_{i}$ is a Banach contraction, then $\lambda_{1} T_{1}+\cdots+\lambda_{n} T_{n}$ is a Banach contraction.

Corollary 4.11 (backward-backward iteration) Let $A_{1}$ and $A_{2}$ be two maximally monotone operators from $X$ to $X$, and assume that one of these is disjointly injective. Then the (backwardbackward) composition $T_{1} T_{2}$ is strictly nonexpansive.

Proof. Combine Theorem 2.1](ix) and Lemma 4.10.

Corollary 4.12 (Douglas-Rachford iteration) Let $A_{1}$ and $A_{2}$ be two maximally monotone operators from $X$ to $X$, and assume that one of these is both at most single-valued and strictly monotone (as is, e.g., the subdifferential operator of a Legendre function; see Corollary [3.9). Denote the resolvents of $A_{1}$ and $A_{2}$ by $T_{1}$ and $T_{2}$, respectively. Then the operator governing the Douglas-Rachford iteration, i.e.,

$$
T:=\frac{1}{2}\left(2 T_{1}-\mathrm{Id}\right)\left(2 T_{2}-\mathrm{Id}\right)+\frac{1}{2} \mathrm{Id},
$$

is not just firmly nonexpansive but also strictly nonexpansive; consequently, Fix $T$ is either empty or a singleton.

Proof. In view of Theorem 3.5 and Theorem 4.1, we see that $2 T_{1}-\mathrm{Id}$ and $2 T_{2}-\mathrm{Id}$ are both nonexpansive, and one of these two is strictly nonexpansive. By Lemma 4.10.(iii), $\left(2 T_{1}-\mathrm{Id}\right)\left(2 T_{2}-\mathrm{Id}\right)$ is strictly nonexpansive. Hence, by Lemma 4.10(iv), $T$ is strictly nonexpansive. 
Remark 4.13 Consider Corollary 4.12, and assume that $A_{i}$, where $i \in\{1,2\}$, satisfies condition (i) in Corollary 4.5. Then $2 T_{i}-$ Id is a Banach contraction by Corollary 4.5 . Furthermore, Lemma 4.10 now shows that the Douglas-Rachford operator $T$ defined in (50) is a Banach contraction. Thus, Fix $T$ is a singleton and the unique fixed point may be found as the strong limit of any sequence of Banach-Picard iterates for T. See also [5], Section 25.2] for various variants and strengthenings.

\section{Acknowledgments}

Heinz Bauschke was partially supported by the Natural Sciences and Engineering Research Council of Canada and by the Canada Research Chair Program. Xianfu Wang was partially supported by the Natural Sciences and Engineering Research Council of Canada.

\section{References}

[1] J.-B. Baillon and G. Haddad, Quelques propriétés des opérateurs angle-bornés et ncycliquement monotones, Israel Journal of Mathematics 26 (1977), 137-150.

[2] S. Bartz, H.H. Bauschke, J.M. Borwein, S. Reich, and X. Wang, Fitzpatrick functions, cyclic monotonicity and Rockafellar's antiderivative, Nonlinear Analysis 66 (2007), 1198-1223.

[3] H.H. Bauschke, J.M. Borwein, and P.L. Combettes, Essential smoothness, essential strict convexity, and Legendre functions in Banach spaces, Communications in Contemporary Mathematics 3 (2001), 615-647.

[4] H.H. Bauschke and P.L. Combettes, The Baillon-Haddad theorem revisited, Journal of Convex Analysis 17 (2010), 781-787.

[5] H.H. Bauschke and P.L. Combettes, Convex Analysis and Monotone Operator Theory in Hilbert Spaces, Springer-Verlag, 2011.

[6] J.M. Borwein and J.D. Vanderwerff, Convex Functions, Cambridge University Press, 2010.

[7] H. Brézis, Operateurs Maximaux Monotones et Semi-Groupes de Contractions dans les Espaces de Hilbert, North-Holland/Elsevier, 1973.

[8] F.E. Browder, Convergence theorems for sequences of nonlinear operators in Banach spaces, Mathematische Zeitschrift 100 (1967), 201-225.

[9] R.S. Burachik and A.N. Iusem, Set-Valued Mappings and Enlargements of Monotone Operators, Springer-Verlag, 2008. 
[10] Y. Censor, A.N. Iusem, and S.A. Zenios, An interior point method with Bregman functions for the variational inequality problem with paramonotone operators, Mathematical Programming Series A 81 (1998), 373-400.

[11] R. Cross, Multivalued Linear Operators, Marcel Dekker, 1998.

[12] F. Deutsch, Best Approximation in Inner Product Spaces, Springer-Verlag, 2001.

[13] J. Eckstein and D.P. Bertsekas, On the Douglas-Rachford splitting method and the proximal point algorithm for maximal monotone operators, Mathematical Programming Series A 55 (1992), 293318.

[14] L. Elsner, I. Koltracht, and M. Neumann, Convergence of sequential and asynchronous nonlinear paracontractions, Numerische Mathematik 62 (1992), 305-319.

[15] K. Goebel and W.A. Kirk, Topics in Metric Fixed Point Theory, Cambridge University Press, 1990.

[16] K. Goebel and S. Reich, Uniform Convexity, Hyperbolic Geometry, and Nonexpansive Mappings, Marcel Dekker, 1984.

[17] G.J. Minty, Monotone (nonlinear) operators in Hilbert spaces, Duke Mathematical Journal 29 (1962), 341-346.

[18] J.-J. Moreau, Proximité et dualité dans un espace hilbertien, Bulletin de la Société Mathématique de France 93 (1965), 273-299.

[19] R.T. Rockafellar, Convex Analysis, Princeton University Press, Princeton, 1970.

[20] R.T. Rockafellar, Monotone operators and the proximal point algorithm, SIAM Journal on Control and Optimization 14 (1976), 877-898.

[21] R.T. Rockafellar and R. J-B Wets, Variational Analysis, Springer-Verlag, 1998.

[22] S. Simons, Minimax and Monotonicity, Springer-Verlag, 1998.

[23] S. Simons, From Hahn-Banach to Monotonicity, Springer-Verlag, 2008.

[24] C. Zălinescu, Convex Analysis in General Vector Spaces, World Scientific Publishing, 2002.

[25] E.H. Zarantonello, Projections on convex sets in Hilbert space and spectral theory I. Projections on convex sets, in Contributions to Nonlinear Functional Analysis, E.H. Zarantonello (editor), pp. 237-341, Academic Press, 1971.

[26] E. Zeidler, Nonlinear Functional Analysis and Its Applications II/A: Linear Monotone Operators, Springer-Verlag, 1990.

[27] E. Zeidler, Nonlinear Functional Analysis and Its Applications II/B: Nonlinear Monotone Operators, Springer-Verlag, 1990.

[28] E. Zeidler, Nonlinear Functional Analysis and Its Applications I: Fixed Point Theorems, SpringerVerlag, 1993. 